\title{
Cardiovascular Risk Assessment in the Nursing Team of a Cardiology Hospital
}

\author{
Marcela Paulino Moreira da Silva1, Claudinalle Farias Queiroz de Souza², \\ Kelly Cristina Torres Lemes², Dândara Nayara Azevêdo Dantas', \\ Anne Karoline Candido Araújo ${ }^{1}$, Tatiana Maria Nóbrega Elias' ${ }^{1}$, \\ Rejane Maria Paiva de Menezes ${ }^{1}$, Bertha Cruz Enders ${ }^{1}$ \\ ${ }^{1}$ University Center of Rio Grande do Norte, Natal, Brazil \\ ${ }^{2}$ University Center of Pernambuco, Recife, Brazil \\ Email:marcelasmp@hotmail.com, annearaujoenf@hotmail.com,dandara_dantas@hotmail.com, \\ tatianaelias@gmail.com, rejemene@gmail.com, berthacruz.enders@gmail.com, \\ claudinalle.souza@upe.br, kellylemes05@gmail.com
}

Received 15 June 2016; accepted 25 July 2016; published 28 July 2016

Copyright (C) 2016 by authors and Scientific Research Publishing Inc.

This work is licensed under the Creative Commons Attribution International License (CC BY). http://creativecommons.org/licenses/by/4.0/

(c) (i) Open Access

\begin{abstract}
Objective: Cardiovascular diseases are the most common causes of morbidity and the leading cause of mortality in the world scenario, accounting for about $20 \%$ of all deaths in individuals over 30 years. It has attributed this to the increase in the company's exposure to risk factors. It identifies cardiovascular risk factors in the nursing team and compares the cardiovascular risk by Framingham score among professionals of middle and upper working in a referral hospital in cardiology. Method: Cross-sectional quantitative study was conducted in 2014, in a reference hospital in cardiology located in Recife/Pernambuco, Brazil. Data of cardiologic risk factors were collected from 82 nursing team members, comprised of technical, auxiliary nurses, and nurses between 30 and 74 years of age. The cardiovascular risk level was evaluated by the Framingham Score. Data were organized and analyzed by means of the SPSS, with descriptive statistics and Student-t test for the continuous variables. Results: Family history, stress and sedentary life style were the prevalent risk factors in more than half of the professionals. Only $5.23 \%$ of the technical and auxiliary nurses had a high risk score for cardiovascular event. Conclusion: The data indicates a low cardiovascular risk for these professionals and the presence of risk factors that can be modified. Health surveillance of these professionals is necessary in order to avoid a change to a risk of greater vulnerability.
\end{abstract}

\section{Keywords}

Nursing, Risk Factors, Cardiovascular Disease

How to cite this paper: da Silva, M.P.M., de Souza, C.F.Q., Lemes, K.C.T., Dantas, D.N.A., Araújo, A.K.C., Elias, T.M.N., de Menezes, R.M.P. and Enders, B.C. (2016) Cardiovascular Risk Assessment in the Nursing Team of a Cardiology Hospital. Open Journal of Nursing, 6, 541-548. http://dx.doi.org/10.4236/ojn.2016.67057 


\section{Introduction}

Cardiovascular diseases (CVD) are the most common causes of morbidity and the leading cause of mortality in the world scenario, with about $20 \%$ of all deaths in people over 30 years [1]. In Brazil, the CVD were the main causes of mortality in 2007 with $30 \%$ of deaths [2] and in Pernambuco state there were 22,900 hospital admissions from 2008 to 2012 with this problem [3].

This fact is due to the increase in the society exposure to modifiable risk factors such as physical inactivity, smoking, obesity, stress, dyslipidemia, hypertension (SAH), diabetes. It is known that the sum of these factors has an exponential effect on the development of CVD [4] [5].

Although the causal relationship between employment status and CVD has not been well understood, there is the presence of risk factors among health professionals [6] [7]. Nursing, as a category with many professionals in health services, they are a vulnerable group for these diseases [8]. Several factors may contribute to this problem: emotional, double shifts, stay on the institutions and fragile working conditions. Also, physical inactivity and high weight are modifiable factors prevalent in this population [8] [9].

Thus, it is believed that the identification of cardiovascular risk in the nursing team can help in the indication of means and strategies to change their lifestyle and self-care. In this perspective, the objectives of this study are to identify the cardiovascular risk factors in the nursing team and compare the cardiovascular risk by Framingham Score among professionals working in a hospital reference in cardiology.

It is supposed that the environment and work processes are essential elements in the health condition of the workers, as the concept of surveillance in workers' health in the country [10]. Moreover, the relationship between health, environment and work process is an important focus of study for workers' health surveillance.

\section{Method}

The descriptive, exploratory, and cross-sectoral study with a quantitative approach was developed in cardiology sectors of a tertiary hospital, reference in Cardiology, located in the north of the city of Recife, Pernambuco. The population was 114 professionals composed of nurses, technicians and nursing assistants who worked in hemodynamics, coronary care unit, emergency and cardiology ward.

The sampling method was random. Inclusion criteria were professionals working for the state in the statutory regime or Consolidation of Labor Laws (CLT), having daytime work shift and having an age greater or equal to 30 years old and less than 74 years old. This last criterion was included to understand the score requirement of Framingham method that was used to assess the risk factors in this study. Professionals who were on maternity and health leave during the study period were excluded.

Six professionals refused to participate, twenty-three were working exclusively at night, two were on leave and did not have age-defined criteria. Thus, the sample consisted of 82 professionals.

The variables under study were sociodemographic characteristics and related risk factors reported in the literature, such as family history, lifestyle habits, anthropometry of clinical laboratory analysis data, and degree of risk for cardiovascular events.

The instrument used was adapted from an earlier study [11], with closed questions and divided into 6 modules: Module I-It was intended to obtain personal identification data (name, phone number, age, gender, race, marital status, academic degrees); Module II—Family history (data on family history); Module III—Life habits (experiment and/or use of tobacco and alcohol, current health problem, stress and physical inactivity); Module IVAnthropometric data (weight, height, body mass index (BMI), abdominal circumference (AC) and blood pressure (BP); Module V-Laboratory measurements (total cholesterol, High Density Lipoproteins, HDL, Low Density Lipoproteins-LDL, cholesterol, triglycerides and blood glucose).

Module VI consisted of a specific tool for risk stratification of cardiovascular prepared according to the Framingham Score.

The Framingham Score was originated from an American population study called the Framingham study to estimate the risk of a cardiovascular event in the people, considering the sum of the individual clinical characteristics (age, total cholesterol, HDL, BP, diabetes and smoking), classifying the risk as low (below 10\%), medium (10\% to $20 \%$ ) and high (above $20 \%$ ) [12]. Although it has its origin in a US population, the method is recommended to use it in Brazil, due to CVD mortality in the country was similar to the countries where it has been validated and has been used in studies of various populations in the world [13].

To measure the BP, aneroid sphygmomanometers, premium brand were used, calibrated by the National In- 
stitute of Metrology, Standardization and Industrial Quality (INMETRO). BP was measured after ten minutes of rest, in the sitting position, with the left arm supported at heart level. As normal SBP measurement was used 18.5 and or equal to 25 and the obesity was with a BMI greater than $30 \mathrm{~kg} / \mathrm{m}^{2}$ [14].

The WC was measured using an inelastic tape. The measurement was taken in the middle of the distance between the iliac crest and below the costal margin. For reference values, it was classified as increased far above $102 \mathrm{~cm}$ values for men and $88 \mathrm{~cm}$ for women [15].

Data collection took place between July and October 2014 and was carried out through interviews by the researcher with the collaboration of three residents of nursing, properly trained. After prior contact and acceptance to participate in the study, participants answered the questions of the first three modules at a scheduled time. Subsequently, they underwent measurement of BP, BMI, and WC to fill the Module IV data.

At a later stage, a collection of laboratory tests related to module $\mathrm{V}$ were scheduled. The professionals were instructed to perform fasting for 12 hours for measurement of blood glucose, total cholesterol, HDL, LDL, triglycerides.

This collection was performed by a hospital laboratory professional, guided by the researcher. The results of laboratory tests were monitored, with one copy for the participant and one for the researcher.

Data were tabulated and analyzed using Statistical Package for Social Sciences (SPSS) version 20.0 Windows $^{\circledR}$. Continuous variables were expressed as means and standard deviation and evaluated using the Student $\mathrm{t}$ test. Categorical variables were expressed as percentages. A confidence level of $95 \%$, the margin of error of $5 \%$ were adopted and significant values of $\mathrm{p}<0.05$ were considered.

The considered normal reference values were: total cholesterol (TC) $<200 \mathrm{mg} / \mathrm{dl}$, HDL from 40 to $60 \mathrm{mg} / \mathrm{dl}$, LDL from 100 to 129 triglycerides < $150 \mathrm{mg} / \mathrm{dl}$ and glucose 65 to $99 \mathrm{mg} / \mathrm{dl}$. Any other result and over these values were considered abnormal.

The percentage risk of developing acute coronary disease through the score table of Framingham was calculated for all professionals as low $(<10 \%)$, medium $(10 \%$ to $<20 \%)$ and high risk $(\geq 20 \%)[5]$.

After obtaining the results, the professionals were guided individually about their cardiovascular risk, through nursing prescriptions and guidelines about healthy eating habits. The food that should be avoided and those healthier were indicated.

The study was approved by the Research Ethics Committee at the study site, with opinion $\mathrm{N}^{\circ} \mathrm{CAAE}$ : 32557114.7.0000.5197 and complies with Resolution 466/2012 of the National Health Council governing research involving human beings. The participants signed a free and informed consent form (TCLE) and were informed about the confidentiality of information and the relevance of the topic.

\section{Results}

There were 82 professional evaluated, $47.50 \%(n=39)$ were nursing technicians, 30.50\% $(n=25)$ were nurses and $22.00 \%(n=18)$ were nursing assistants. For the presentation of the results found, these professionals were divided into two groups: Group A-mid-level professionals (technicians and nursing assistants $/ \mathrm{n}=57$ ); Group $\mathrm{B}$-higher-level professionals (nurses $/ \mathrm{n}=25$ ). Data regarding socio-demographic characteristics of these groups are shown in Table 1. Also, the mean age was $45.77( \pm 7.23)$ years old for group A and $46.20( \pm 9.43)$ years old for the group $B(p=0.08)$.

From Figure 1, it can be seen that most risk factors were present in group B formed by nurses. However, the stress factors, physical inactivity, and family history have been identified in more than $50 \%$ in both groups. Stress was configured as the most prevalent risk factor among nurses (Group B), with a percentage of $90 \%$.

Concerning clinical, laboratory and anthropometric of the groups shown in Table 2, the technicians and assistants (Group B) had a higher mean of BMI, diastolic blood pressure (DBP) and HDL cholesterol fraction when compared to the Group of nurses. There was a statistically significant difference between the HDL fraction between the groups $(p=0.02)$. However, the group of nurses showed levels of systolic blood pressure (SBP), Abdominal Circumference (AC) in women, blood glucose, total cholesterol (TC), LDL cholesterol and triglycerides with higher average compared to the other group. The parameters changed in both groups were AC, TC, LDL, and Triglycerides.

Although some measures are changed to values in both groups, the high cardiology risk in the risk stratification analysis according to the Framingham Score was present in only $5.23 \%(n=3)$ of the mid-level professionals and none in the higher level professionals, predominantly with low risk (Figure 2). 


\section{Discussion}

The results found a predominance of females with a proportion of the characterization of the sociohistorical context of the profession, originated culture in nursing. The feminization in activities involving the treatment and care of people is considered a structural feature of the health sector activities, particularly about nursing [1] [8] [16].

As the clinical situation related to gender, studies have shown that males have a higher predisposition to de-

Table 1. Socio-demographic characteristics and work on the nursing team of a cardiology hospital, according to the professional level. Recife, PE, Brazil, $2014(\mathrm{~N}=82)$.

\begin{tabular}{|c|c|c|c|}
\hline \multicolumn{2}{|c|}{ VARIABLES } & Group A & Group B \\
\hline \multirow{2}{*}{ Gender } & Male & $46(80.70)$ & $23(92.00)$ \\
\hline & Female & 11 (19.29) & $2(8.00)$ \\
\hline \multirow{5}{*}{ Race } & White & 22 (38.59) & $7(28.00)$ \\
\hline & Brown & $17(29.82)$ & $14(56.00)$ \\
\hline & Black & $14(24.56)$ & $3(12.00)$ \\
\hline & Mulatto & $3(5.26)$ & $1(4.00)$ \\
\hline & Indigenous & $1(1.75)$ & $0(0.00)$ \\
\hline \multirow{5}{*}{ Marital status } & Married & 22 (38.59) & $15(60.00)$ \\
\hline & Single & $16(28.07)$ & $3(12.00)$ \\
\hline & Divorced & $10(17.54)$ & $6(24.00)$ \\
\hline & Stable union & $8(14.03)$ & $1(4.00)$ \\
\hline & Widow & $1(1.75)$ & $0(0.00)$ \\
\hline \multirow{2}{*}{ Family income monthly } & 1 to 3 minimum wage ${ }^{*}$ & 33 (57.89) & $1(4.00)$ \\
\hline & 3 to 5 minimum wage & $24(42.10)$ & $24(96.00)$ \\
\hline \multirow{2}{*}{ Children } & Yes & $41(71.92)$ & $18(72.00)$ \\
\hline & No & $16(28.07)$ & $7(28.00)$ \\
\hline \multirow{4}{*}{ Working area } & Infirmary & $23(40.35)$ & $8(32.00)$ \\
\hline & Coronary unit & 17 (29.82) & $9(36.00)$ \\
\hline & Emergency Cardiology & $13(22.80)$ & $6(24.00)$ \\
\hline & Hemodynamics & $4(7.01)$ & $2(8.00)$ \\
\hline
\end{tabular}

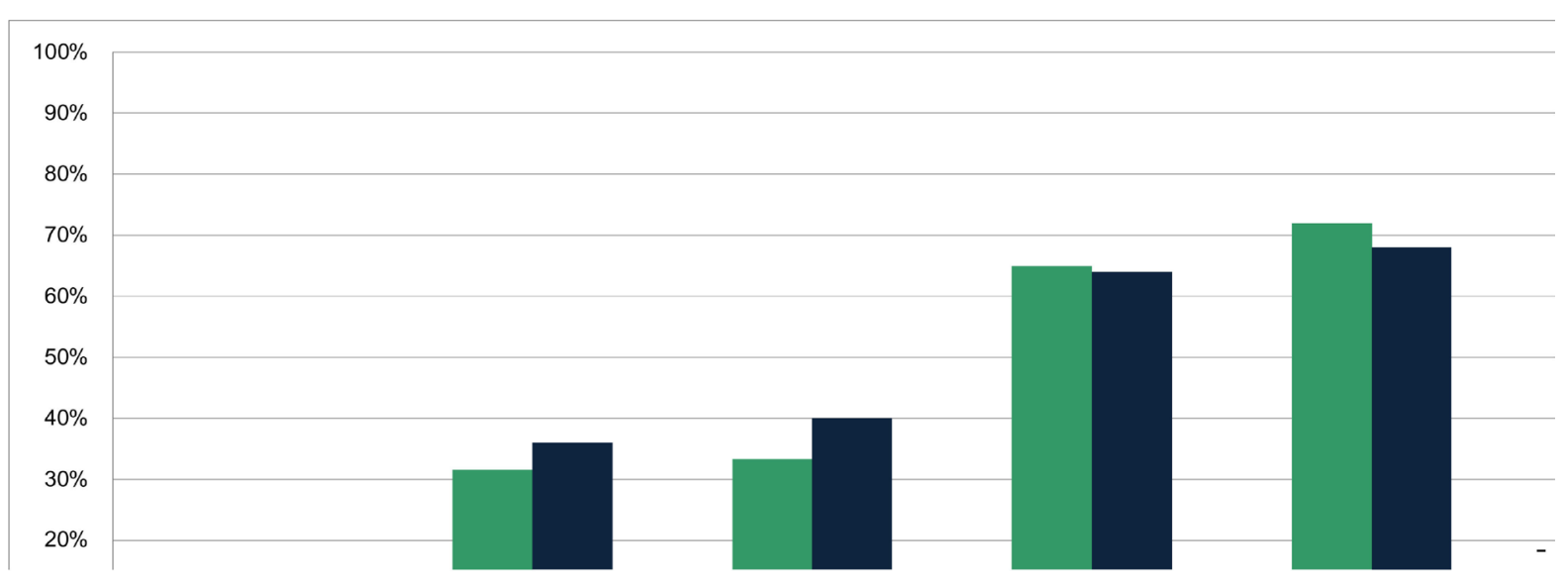

*Group A - mid-level professional $(n=57)$; $†$ Group B - higher level professional $(n=25)$. 1-Tobacco; 2- Obesity; 3-Overweight; 4-Family Background; 5- Physical inactivity; 6- Stress

Figure 1. Prevalence of the main cardiovascular risk factors in the nursing staff of a cardiology hospital, according to the professional level. Recife, Brazil, 2014 ( $n=82)$. 
Table 2. Clinical, laboratory and anthropometric characteristics of the nursing staff of a cardiology hospital, according to professional category. Recife/PE, Brazil, $2014(\mathrm{n}=82)$.

\begin{tabular}{ccccc}
\hline Variables & Reference values & Group A & Group B & P \\
\hline SBP & $<130 \mathrm{mmHg}$ & $119.52 \pm 15.12$ & $118.92 \pm 10.45$ & 0.14 \\
DBP $^{\dagger}$ & $<85 \mathrm{mmHg}$ & $71.89 \pm 14.65$ & $74.48 \pm 8.65$ & 0.34 \\
BMI $^{\ddagger}$ & $18.5 \mathrm{a} 25 \mathrm{~kg} / \mathrm{m}^{2}$ & $25.55 \pm 4.53$ & $28.86 \pm 5.04$ & 0.65 \\
AC $^{\S}$ & & & & 0.36 \\
Male & $\leq 94 \mathrm{~cm}$ & $95.80 \pm 6.44$ & $98.00 \pm 12.72$ & \\
Female & $\leq 80 \mathrm{~cm}$ & $98.89 \pm 9.18$ & $85.90 \pm 3.60$ & \\
Glycemia & $65 \mathrm{a} 99 \mathrm{mg} / \mathrm{dL}$ & $98.98 \pm 28.92$ & $89.64 \pm 9,19$ & 0.08 \\
Total Cholesterol & $200 \mathrm{mg} / \mathrm{dL}$ & $210.45 \pm 43.33$ & $188.40 \pm 35.66$ & 0.50 \\
HDL// & $40-60 \mathrm{mg} / \mathrm{dL}$ & $53.52 \pm 11.36$ & $55.56 \pm 7.63$ & 0.02 \\
LDL & $100-129 \mathrm{mg} / \mathrm{dL}$ & $131.10 \pm 41.80$ & $116.96 \pm 29.42$ & 0.13 \\
Triglicerídeos & $<150 \mathrm{mg} / \mathrm{dL}$ & $129.13 \pm 60.20$ & $110.88 \pm 42.63$ & 0.05 \\
\hline
\end{tabular}

${ }^{*}$ SBP—Systolic Blood Pressure; ${ }^{\dagger} \mathrm{DBP}$ —Diastolic Blood Pressure; ${ }^{\ddagger} \mathrm{BMI}$ —Body Mass Index; ${ }^{\S} \mathrm{CA}$ —Abdominal Circumference; //HDL-High-Density Lipoproteins; ${ }^{\circledR}$ LDL-Low-Density Lipoproteins.

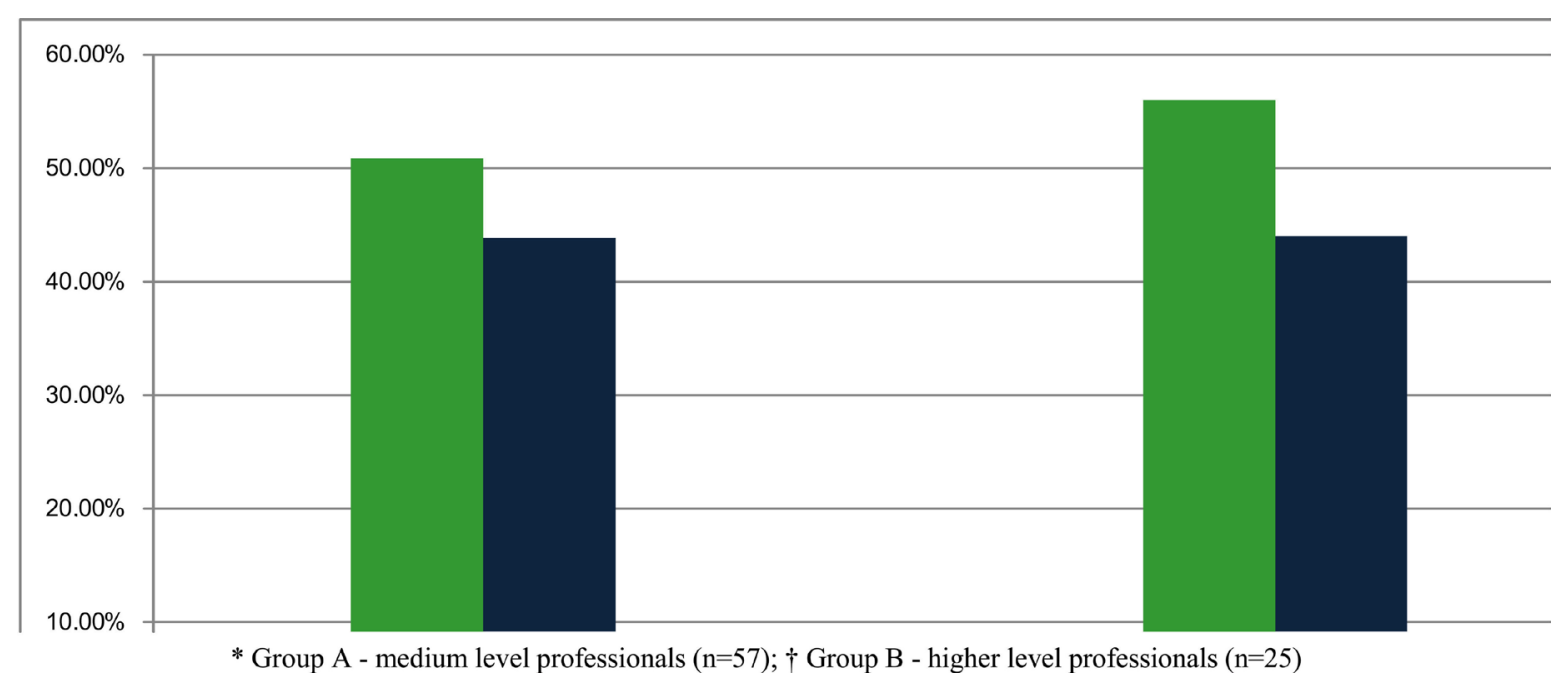

Figure 2. Stratification of cardiovascular risk estimated by the Framingham Score in the nursing staff of a cardiology hospital, according to professional category. Recife/PE, Brazil, $2014(\mathrm{n}=82)$.

velop heart disease. Although, other studies highlight that there is an increased risk for post-menopausal women due to hormonal fluctuations to which they are exposed during this period [1]-[9].

There was a predominance of mid-level professionals, especially in the category of nursing staff due to the higher amount of the team belong to this category. Data from the Federal Nursing Council revealed that in 2010, there were $19.8 \%$ nurses, $43.2 \%$ nursing technicians, and 36.8\% nursing assistants, registered with the largest amount of mid-level professionals [16].

Regarding the age, the data showed that both mid-level or higher level professionals had an average age of 46 years old. It is known that advancing age by itself, increases the risk of developing CVD and should not be viewed solely as a purely biological factor, deserving more effective preventive and educational measures for the factors that can be modified [9].

Previous studies indicate that the risk of CVD is doubled across the years of the individual, due to longer exposure to risk factors. Advancing age is a marker of some atherosclerotic plaques. The greater the amount, the greater the risk of ischemic cardiovascular disease [17] [18].

Among the Cardiovascular Risk Factors, it was noticed that most of the subjects had family history who were sedentary and non-smokers, corroborating the authors [9] when referring to the prevalence of these risk factors 
in the population, in addition to a high weight [9].

Still on the CRF on the level of stress, most of the nursing staff had a moderate level of stress, but there was a higher prevalence in the group of nurses. Similar results were found in a study conducted in hospitals of the state of Paraná Western region which showed nurses with an average stress level [19].

Findings in the literature show that this fact can be attributed to unhealthy working conditions, domestic overhead and monthly income, in addition to activities that require greater responsibility. Nurses perform management activities with the planning of nursing care, personal management, the prescription of nursing care assuming all the greater technical complexity of care and direction of nursing services [20] [21].

Regarding the clinical characteristics and modifiable risk factors among mid-level and higher professionals, the findings showed that for the BP, professionals had an average of SBP and DBP, normotensive in both groups, not consistent with data presented in a study revealing a high prevalence of hypertension in nurses [22].

However, in the BMI, average above the recommended by ABESO with overweight and obesity were more prevalent in higher level professionals, contrasting results found in the study conducted at the University Hospital of the Federal University of Santa Maria who showed no statistically significant difference between nursing professionals [23].

According to the Brazilian Association for Study of Obesity (ABESO), BMI is a good indicator, but not totally related to body fat, because it has some limitations, such as it does not distinguish between fat mass of lean mass, it does not necessarily reflect the distribution of body fat, and it does not necessarily indicate the same level of fat in different populations. Nevertheless, the combination of BMI and fat distribution measures can help solving some problems of using only the BMI [15].

Therefore, research on WC was also conducted, which showed that both groups presented with threshold values when considering the standard deviation, becoming problematic for the existing potential risk. However, the average level of female professionals showed a high average in this measurement [15].

Studies show the need to adhere to preventive measures for the control of obesity, overweight, and reduced BMI because the fat deposits in the abdomen was a strong predictor for CVD. To achieve this goal, it is necessary to strike a balance in eating habits continuously to achieve success, and the acquisition of modest interventions in lifestyle to reduce cardiovascular risk [9]-[24].

On glycemic variables, the HDL fraction, and triglycerides, results showed levels within normal parameters in both groups. However, the evaluation of TC and LDL showed that most of the mid-level professionals presented above normal levels, while most of the higher-level results were within the limits. This may be related to socioeconomic factors such as income and education. By having, in general, monthly income below the higher-level professionals, nursing technicians, and assistants often need to work in two or more institutions to meet their daily needs, thus causing acquisition of bad eating habits or inferior quality, and not engaging in physical activity [25].

Thus, these factors had a significant correlation for CVD, corroborating similar study conducted in the city of Juazeiro do Norte/CE [11].

As for risk stratification by the Framingham Score, a low risk was observed for most professionals, both mid-level as higher. However, most individuals considered at risk for ten years may change the score to a high risk over a lifetime. This can occur, especially if some risk factors existing today, become sets, as shown by the Brazilian guideline of dyslipidemia and prevention of atherosclerosis; that is the cardiovascular risk is dynamic if preventive measures are not adopted in daily life [15]-[26].

The current discussion on the impact of CVD and its risk factors supports this study because they are situations to which workers are likely, whether at work, for food education, and in leisure. They are typical circumstances of time in which the practicality, versatility, and pragmatism prevail, mainly driven by the new conditions of work abilities [11] [27] [28].

\section{Conclusions}

The study reveals the need for preventive strategies and healthy habits need to be incorporated into the development policy of human resources in the hospital to implement the health surveillance of these workers and to encourage self-care.

It is found that a sedentary lifestyle and family histories are risk factors most prevalent in mid-level nursing professionals and smoking, obesity and overweight are more prevalent in the group of higher-level professionals. 
Although most professionals in this study, both of the mid and higher level, be at low risk for a future cardiovascular injury, there is a significant quantitative with medium risk.

Thus, the results indicate the need for preventive strategies and healthy habits to be incorporated into the development policy of human resources in the hospital to implement the health surveillance of these workers and to encourage self-care. Professionals as entrepreneurs of their well-being may carry specific group changes to meet the demands of their working environment.

Therefore, it is suggested a partnership between those involved in the care and management for the construction of environments in service for the workplace exercise to encourage professionals to this habit as a way to reduce a possible cardiovascular event. Also, there is the importance of an active continuing education within the workplace to discuss with the team on the theme showing the need to adhere to reducing actions of existing or future risk for cardiovascular disease.

It must be mentioned that this study focuses on the cardiovascular risk factors in its relationship with the professional work context. However, it does not evaluate other factors that may also be associated with this vulnerability, as the existence of other jobs, the level of satisfaction with work, family demands, among others, which can be configured as a limitation of the study. More comprehensive studies will further elucidate the relationship between health professionals and the cardiology hospital setting.

\section{References}

[1] Mansur, A.P. and Favarato, D. (2012) Mortality from Cardiovascular Diseases in Brazil and in the Metropolitan Region of São Paulo: Update 2011. Arquivos Brasileiros de Cardiologia, 99, 755-761. http://dx.doi.org/10.1590/S0066-782X2012005000061

[2] Goulart, F.A.A. (2011) Chronic Non-Communicable Diseases: Control Strategies and Challenges and to the Health System. Ministry of Health, Brasilia.

[3] Mendonça, S.S. and Albuquerque, E.C. (2014) Profile of Hospitalizations for Sensitive Conditions Primary Care in Pernambuco, 2008 to 2012. Epidemiologia e Serviços de Saúde, 3, 463-74.

[4] Martins, L.N., Souza, L.S., Silva, C.S., Machado, R.S., Silva, C.E.F. and Vilagra, M.M. (2011) Prevalence of Cardiovascular Risk Factors in Adults Admitted to the Chest Pain Unit in Vassouras, RJ. Revista Brasileira de Cardiologia Invasiva, 24, 299-307.

[5] Cavagiono, L. and Pierin, A.M.G. (2012) Cardiovascular Risk in Health Professional Pre-Hospital Care Services. Revista da Escola de Enfermagem da USP, 46, 395-493.

[6] Keys, C.S., Piglet, M.P.C., Braga Junior-ACR and Sirino, A.C.A. (2015) Identification of Risk Factors for Cardiovascular Disease among Health Professionals. Arq. Ciênc. Health, 22, 39-47.

[7] Hwang, W.J. and Hong, O. (2012) Work-Related Cardiovascular Disease Risk Factors Using the Socioecological Approach: Implications for Practice and Research. European Journal of Cardiovascular Nursing, 11, 114-126. http://dx.doi.org/10.1177/1474515111430890

[8] Oliveira, A.S.S. (2011) Quality of Life and Cardiovascular Risk Factors Modifiable Nurses in Family Health Strategy. Dissertation, State University of Ceará, Fortaleza.

[9] Magellan, F.J., Mendonça, L.B.A., Rebouças, C.B.A., Lima, F.E.T., Custódio, I.L. and Oliveira, S.C. (2014) Risk Factors for Cardiovascular Disease among Nursing Professionals: Health Promotion Strategies. Revista Brasileira de Enfermagem, 67, 394-400.

[10] Brazil. Ministry of Health.Protocol No. 008/2011 (2011) Establishes the Guidelines of the National Policy of Promoting Health of the Health System Employee-SUS. Ministry of Health, Brasilia.

[11] Gomes, E.B. (2010) Analysis of Cardiovascular Risk in Young Adults of School Juazeiro of Ceara North. Dissertation, State University of Ceará/UEC, Ceará.

[12] Dawber, T.R. (1980) The Framingham Study. The Epidemiologic of Atherosclerotic Disease. Harvard University Press, Cambridge. http://dx.doi.org/10.4159/harvard.9780674492097

[13] Petterle, W.C. and Polanczyk, C.A. (2011) Critical Evaluation of Risk Scores. Rev Soc Cardiol Rio Gd South, 19, 1-6.

[14] Brazilian Cardiology Society, Brazilian Hypertension Society, Brazilian Society of Nephrology (2010) VI Brazilian Guidelines on Hypertension. Arquivos Brasileiros de Cardiologia, 95, 1-51.

[15] Brazilian Association for the Study of Obesity and Metabolic Syndrome-ABESO (2009) Brazilian Guidelines on Obesity. 3rd Edition, AC Pharmaceuticals, Itapev.

[16] Machado, M.H., Vieira, A.L.S. and Oliveira, E. (2012) Construindo o perfil da enfermagem. Enfermagem em Foco, 3, 119-122. 
[17] Kalaycıoğlu, G.T., Ayka, B.C., Boyaci, F., Turhan, T. and Gül, I. (2014) Value of Coronary Calcium Score to Predict the Severity or Complexity of Coronary Artery Disease. Arquivos Brasileiros De Cardiologia, 102, 120-127.

[18] Muniz, L.C., Schneider, B.C., Silva, I.C.M., Matijasevich, A. and Santos, I.S. (2012) Accumulated Behavioral Risk Factors for Cardiovascular Diseases in Southern Brazil. Revista de Saúde Pública, 46, 534-542. http://dx.doi.org/10.1590/S0034-89102012005000021

[19] Freire, C.M.V. (2012) Cardiovascular Risk Markers: A Lot of Information and Uncertainty. Revista Brasileira Ecocardiogr Imagem Cardiovasc, 25, 260-261.

[20] Inoue, K., Versa, G.L.G., Murassak, A.C.Y., Melo, W.A. and Matsuda, L.M. (2013) Occupational Stress in Intensive Care Nurses Who Provide Direct Care to Critical Patients. Revista Brasileira de Enfermagem, 66, 722-729. http://dx.doi.org/10.1590/S0034-71672013000500013

[21] Costa, D.T. and Martins, C.M.F. (2011) Stress in Nursing Professionals: Impact of the Conflict in the Group and Medical Power. Revista da Escola de Enfermagem da USP, 45, 1191-1198.

[22] Souza, I.S.N., Silva, F.J., Gomes, R.L.V. and Frazão, I.S. (2013) Stressful Situations Work of Nurses at a School Hospital. Revista de Enfermagem da UFSM, 3, 287-295.

[23] Custódio, I.L., Lima, F.E.T., Almeida, M.I., Silva, L.F. and Monteiro, A.R.M. (2011) Socio-Demographic and Clinical Profile of a Nursing Team Carries Hypertension. Revista Brasileira de Enfermagem, 64, 18-24. http://dx.doi.org/10.1590/S0034-71672011000100003

[24] Toledo, M.T.T., Abreu, M.N. and Lopes, A.C.S. (2013) Adherence to Healthy Lifestyles through Counseling by Health Professionals. Revista de Saúde Pública, 47, 540-548. http://dx.doi.org/10.1590/S0034-8910.2013047003936

[25] Pires, C.G.S. (2013) Cardiovascular Risk Factors among Student (the) Nursing the First and Last School Years. Thesis, School of Nursing of the Federal University of Bahia, Salvador.

[26] Mafra, F. and Oliveira, H. (2008) Evaluation of Cardiovascular Risk-Methodologies and Their Implications for Clinical Practice. Rev Port Clin General, 24, 391-400.

[27] Garden, T.H., Souza, A.L.L., Povoa, T.R., Barroso, W.S., Chinem, B. and Garden, P.C.V. (2014) Comparison of Cardiovascular Risk Factors in Different Areas in Health Twenty Years Interval. Arquivos Brasileiros de Cardiologia, 103, 493-501.

[28] Nobre, M.R.C., Domingues, R.Z.L., Silva, R., Colugnati, F.A.B. and Taddei, A.A.C.T. (2006) Prevalence of Overweight, Obesity and Lifestyle Associated with Cardiovascular Risk in Primary School Students. Revista da Associação Médica Brasileira, 52, 118-124. http://dx.doi.org/10.1590/S0104-42302006000200023

\section{Submit or recommend next manuscript to SCIRP and we will provide best service for you:}

Accepting pre-submission inquiries through Email, Facebook, LinkedIn, Twitter, etc.

A wide selection of journals (inclusive of 9 subjects, more than 200 journals)

Providing 24-hour high-quality service

User-friendly online submission system

Fair and swift peer-review system

Efficient typesetting and proofreading procedure

Display of the result of downloads and visits, as well as the number of cited articles

Maximum dissemination of your research work

Submit your manuscript at: http://papersubmission.scirp.org/ 